\title{
Editorial note to: \\ F. Zwicky \\ The redshift of extragalactic nebulae
}

\author{
Jürgen Ehlers
}

Published online: 18 November 2008

(C) Springer Science+Business Media, LLC 2008

Keywords Galaxies: redshift · Dark matter · Cosmology · Golden Oldie

\section{Editor's note}

By Jürgen Ehlers (deceased May 20, 2008)

The important, lasting result of Fritz Zwicky's early paper on extragalactic astrophysics, which is not mentioned in the abstract, is his conclusion, derived in Sect. 5, that the universe contains much more dark than luminous matter. This result is embedded in a review of what was known about "nebulae" and their redshift in 1933. The first methods to determine distances of galaxies and their distribution on the sky and in space are summarized, and explanations for their redshift are considered. In this note I shall make remarks on the changes of quantitative data and comment on the history of the theory of extragalactic redshifts and on Zwicky's attempt to relate the just discovered cosmic radiation to those redshifts.

The distance and diameter of the Andromeda galaxy, given by Zwicky in Sect. 2A as 900 resp. 42 kly (kilo light years), are based on the Cepheid method used by Hubble. The present values are about 2,500 resp. $100 \mathrm{kly}$. The large "increase" of these values is due to Baade's discovery [1] of two types of Cepheids with different period-luminosity relations, belonging to distinct stellar populations, and Sandage's identification of Hubble's "brightest stars" as HII regions, which are much more luminous than stars [2].

The republication of the original paper in translated version can be found via doi:10.1007/s10714-008-0707-4. 
These and later corrections such as taking into account the Malmquist bias led also to the presently accepted value of $(65 \pm 5) \mathrm{km} \mathrm{s}^{-1} \mathrm{Mpc}^{-1}$ instead of Hubble's $558 \mathrm{~km} \mathrm{~s}^{-1} \mathrm{Mpc}^{-1}$ quoted by Zwicky.

Van Maanen's large proper motions and rotation rates of galaxies reported at the end of Sect. 3 came soon to be regarded as erroneous when Hubble [3] established by reliable observations that rotation speeds as large as claimed by van Maanen do not exist.

Zwicky's discussion, in Sect. 4, on "speculations concerning the redshift" illustrates well the difference between the real history of science and what is made of it in textbooks. Zwicky finds none of these theories satisfactory. Explanations based on GRT occur side by side with "tired light" ideas, although the latter had no sound basis in physics, whereas ten years earlier the general method of calculating redshifts in arbitrary GR models and its application to cosmology had been given and published independently by Weyl [4] and Lanczos [5]. (Weyl had even estimated in 1923 (!) the Hubble constant $H_{0} \approx 1,000 \mathrm{~km} \mathrm{~s}^{-1} \mathrm{Mpc}^{-1}$, using Slipher's [6] redshifts and Lundmark's [7] distance estimates.) Their method, applied to the expanding world models of Friedmann [8-11], and Lemaitre [12,13] would have given immediately the redshift formulae-with $\Lambda$ - of the present standard model. This expression satisfies Zwicky's conditions stated at the beginning of Sect. 4, apart from points 6 and 8 . Point 8 could be dismissed soon, as reported above. Point 6 was accounted for by Zwicky in Sect. 5, see below. The main reason for scepticism as regards the GR-based explanations was, I think, that the general explanation of redshifts, particularly their dependence on the metric and the motions of observer and sources, was not understood by astronomers, as is clear from the then still persisting confusion about redshift in de Sitter cosmologies (plural) based on de Sitter's spacetime (singular).

The argument for dark matter in Sect. 5 still stands, apart from numerical values.

The speculations on "penetrating", i.e. cosmic, radiation, in Sect. 6, seem no longer of interest since too little was known about the subject in 1933. The remark on total energy loss of photons, however, underlines the lack of understanding of redshift at the time, at least among astronomers.

\section{Fritz Zwicky: a brief biography}

By Andrzej Krasiński, translated and compiled from Refs. [14,15] below.

Fritz Zwicky was born on 14 February 1898 in Varna (today Bulgaria, then Ottoman Empire), where his father was a trade representative of Swiss textile factories and businessmen. He moved to Switzerland at the age of 6 , to attend a primary school and a high school in Mollis (Glarus canton). He completed his school education at the Industry School (today Mathematisch-Naturwissenschatliches Gymnasium) in Zürich, and then studied mathematics and physics at the Eidgenössische Technische Hochschule (ETH) in Zürich. He graduated under the supervision of Hermann Weyl. He got his $\mathrm{PhD}$ there in 1922 (his PhD supervisor was Peter Debye), and initially worked at the ETH as a scientific assistant. While in Zürich, he is reported to have lived door to door with Lenin [15] (but there are no indications that they were personal acquaintances). In 1925 he obtained a fellowship of the Rockefeller Foundation at the California Institute 
of Technology in Pasadena. He would then continue to live in the USA for more than 40 years. His host in Pasadena was Robert Millikan, who expected Zwicky to work on quantum mechanics of atoms and metals. Zwicky, however, became more interested in astronomy and astrophysics. He carried out astronomical observations at the observatories on Mt Wilson and Mt Palomar. During his lifetime, he discovered 123 supernovae.

During World War 2 he worked for civil defense, studied rocket technology (in 1943-1949 he was the scientific director of the rocket company Aerojet) and collaborated in creating a practical help program for scientific libraries damaged by war. He obtained several patents for his work on jet propulsion engines and fuels. After the war he was sent to Germany and Japan to help re-establish science in those countries. For his activities he was awarded the American "Medal of Freedom" in 1949. He contributed essentially to the 1957 shot of the first human-made bullet into space, an object manufactured on the Earth which should leave the gravity of the Earth for ever. These experiments were done with German V2 rockets, which, when reaching the highest point, would shoot a bullet away.

He is credited with inventing a method of management called the "morphological method" $[14,15]$. One of the ideas of the method is to systematically search for a solution of a problem by trying out all combinations in a matrix of expressions. After the war he tried (unsuccessfully) to introduce this method in the Swiss national defense system.

In 1972, for his work in astronomy, he received a gold medal of the Royal Astronomical Society in London.

He was the author of more than 500 publications, among them the books "Morphological Astronomy" (1957), "Discovery, invention, research in a morphological world image" (in German, 1966) and "Everybody a genius" (in German, 1971). Several cosmic objects and technical inventions are named after him.

In his Swiss years, Zwicky became a mountain climber. Together with his friend Thadeus Reichstein he did some first ascents of new routes in the Alps, some of which were included in the guidebooks of the Swiss Alpine Club (for example, the North Face of Glärnisch). His reason for choosing Pasadena as his residence: "because there are mountains there" [15]. In the winter, Zwicky often would take his skis to his work place in order to jump on a self built ramp near the telescope on Mount Wilson.

The author of Ref. [15] wrote the following: "During the thirties and forties, many of Zwicky's colleagues thought of Zwicky as a 'big mouth', but later generations of astrophysisics should think of him as a creative genius (Thorne). Aversions against Zwicky's habit, 'to call the child by the name' and to consider 'humbleness a lie' survive until now."

Some further quotations from Ref. [15]:

"In the halls of the Physics building at Caltech, Zwicky used to start a conversation to students, whose name he did not know with the words: 'Who the devil are you?' "

"Zwicky used to call other astronomers at the Mount Wilson observatory 'Spherical bastards'. Why spherical? 'Because they were bastards, when looked at from any side'."

"In the sixties, Zwicky had the idea to influence the fission mechanism in the Sun through bombardment from the Earth. The aim was to change the path of the Sun and 
of the whole solar system. He wrote that it should be possible [in this way] to travel to other stars, for example to the neighbouring star-system Alpha Centauri-during a 2,500-year long travel."

He died on 8 February 1974 in Pasadena, but his grave is in Mollis (Glarus canton, Switzerland). His legacy is managed by the "Fritz Zwicky Foundation" (created in Switzerland by the local government of the Glarus canton and the town Mollis) together with the Glarus state library.

More information about Zwicky can be found in the works cited in Refs. [14,15].

\section{References}

\section{Editor's note}

1. Baade, W.: A revision of the extra-galactic distance scale. Trans. IAU 8, 397-398 [reprinted with commentary in Lang, K.R., Gingerich, O. (eds.), A Source Book in Astronomy and Astrophysics, 1900-1975 (Harvard University Press, Cambridge, MA, 1979), 750-752] (1952)

2. Humason, M.L., Mayall, N.U., Sandage, A.R.: Redshifts and magnitudes of extragalactic nebulae. Astron. J. 61, 97 [reprinted in the centennial edition, Astrophys. J. 525C, 640 (1999) with a modern commentary by Paul W. Hodge; also excerpted with commentary in A Source Book in Astronomy and Astrophysics, 1900-1975 edited by Lang, K. R. and Gingerich, O. (Harvard University Press, Cambridge, MA, 1979), 753-762] (1956)

3. Hubble, E.: Angular rotations of spiral nebulae. Astrophys. J. 81, 334-35 (1935)

4. Weyl, H.: On the general relativity theory. Phys. Z. 24, 230-232 (1923)

5. Lanczos, K.: Über die Rotverschiebung in der DeSitterschen Welt. Z. Phys. 17, 168 (1923)

6. Slipher, V.M.: Lowell Obs. Bull. 2, 2.56-2.57 (Bull. No. 58) (1913)

7. Lundmark, K.: Stock. Akad. Hand. $\mathbf{5 0}$ Nr. 8 (1920)

8. Friedmann, A.A.: On the curvature of space. Z. Phys. 10, 377 (1922)

9. Friedmann, A.A.: On the possibility of a world with constant negative curvature of space. Z. Phys. 21, 326 (1924)

10. Friedmann, A.A.: English translation of both papers with historical comments. Gen. Relativ. Gravit. 31, 1985 (1999)

11. Friedmann, A.A.: Addendum. Gen. Relativ. Gravit. 32, 1937 (2000)

12. Lemaître, G.: Un univers homogène de masse constante et de rayon croissant, rendant compte de la vitesse radiale de nébuleuses extra-galactiques, [A homogeneous Universe of constant mass and increasing radius accounting for the radial velocity of the extra-galactic nebulae]. Ann. Soc. Sci. Brux. A47, 49 (1927)

13. Lemaître, G.: A homogeneous Universe of constant mass and increasing radius accounting for the radial velocity of the extra-galactic nebulae. Mon. Not. R. Astr. Soc. 91, 483 (1931)

\section{Fritz Zwicky: a brief biography}

14. http://www.zwicky-stiftung.ch/BioLang.htm

15. Knill, O.: http://www.dynamical-systems.org/zwicky/Zwicky-e.html 